\title{
The relationship between stochastic and temporal dominance under differing instructional sets'
}

\section{PHILIP J. MOHAN, DEPARTMENT OF PSYCHOLOGY, CLAREMONT GRADUATE SCHOOL, Claremont, Calif.}

Forty-four college students were required to make either discrimination or preference judgments under a speed or accuracy orientation. Decision times for the Stochastically Dominant $(S D)$ and Stochastically Non-Dominant (SND) were recorded. The results indicated that the mean $S D$ decision times were significantly faster than the SND decision times under an accuracy orientation. No differences were found between the $S D$ and the SND decision times under a speed set.

There are several mathematical models that describe the relationship between temporal and stochastic dominance in a two-alternative choice situation. ${ }^{2}$ Each model makes a separate prediction as to the nature of the relationship between these two variables, although it has been suggested (Greenberg, 1961; Coombs, 1964) that the perceptual set of the subject may influence this relationship. Specifically, (1) that the mean decision times for the dominant choice should be shorter than the mean decision times for the non-dominant choice under an accuracy set, and (2) that the mean decision times for the dominant choices should be longer than the mean decision times for the nondominant choices under a speed set. These hypotheses were tested in the present study.

Method

The major portion of this experiment is described in its entirety in Greenberg (1961). Forty-four undergraduate students at San Bernardino Valley College served as Ss. The stimuli were six shades of gray, constructed from neutral density filters which were presented in pairwise fashion with the following instructions:

On the screen in front of you will appear two circular patches of gray. You are to choose the patch which appears to be the darker of the two. If it is the one on the left, push the left button; if it is the one on the right, push the right button.

If the $S$ was required to make preferential judgments, the instructions were identical but with the phrase, "you are to choose the patch you prefer as the better representative of a good medium gray," substituted in the second sentence of the above instructions. In order to induce the perceptual sets of speed or accuracy, additional instructions were given. For the $S s$ in the speed group the instructions were as follows: (accuracy instructions are in parentheses)

Try to respond as quickly (accurately) as possible. Although you would like to make correct (rapid) responses, speed (accuracy) is the important and accuracy (speed) is definitely a secondary consideration. Are you ready for the first slide?

The 15 pairs were projected on the screen with the S's choice as well as decision time recorded by the experimenter. Each $S$ made a total of 450 judgments ( 30 replications of each pair) of the stimulus pairs randomized for the order of presentation and counterbalanced for the left-right position of the individual patches.

There were four experimental groups $(N=11)$ since each $S$ made only one type of judgment (discrimination or preference) under one set condition (speed or accuracy).

Results

The differences between the mean dominant and non-dominant decision times were investigated by means of the analysis of variance (Case II, Winer, 1964) with the type of task (Discrimination vs Preference) and set (Speed vs Accuracy) as the uncorrelated variables and dominance (Dominant vs NonDominant) as the correlated dimension.

For the test of the experimental predictions the Sets by Dominance interaction was of primary interest. The fact that it was significant $(F=6.60, p<.025)$ suggests that the relationship between stochastic and temporal dominance is dependent upon the type of instructional set received by the $S$. Further analysis of this interaction revealed a significant difference $(t=8.38, p<.005$; one-tailed) between the means of the dominant and non-dominant decision times $(.970$ vs $1.182 \mathrm{sec})$ under an accuracy set, but no difference $(.389$ vs $.389 \mathrm{sec})$ under a speed set. The significant sets by task interaction $(F=5.44, p<.025)$ was of little interest in the present study. It indicates that the difference between the speed and accuracy decision times is dependent upon the type of task required of the $S$.

Discussion

On the basis of the statistical analyses performed, the following conclusions were drawn: (a) The instructional sets of speed and accuracy differentially affected the relationship between temporal and stochastic dominance. This finding suggests that mathematical models of choice must be modified in such a way to account for the type of perceptual set received by the S. Falmagne (1965) has considered this possibility. (b) Under an accuracy set, the SD decision times were significantly faster than those associated with the SND choices. In this instance, at least, temporal and stochastic dominance are intimately related. This finding provides support for the first prediction and is consistent with the results of earlier experiments (Coombs, 1958; Kellogg, 1931; Petrussic, 1966; and Greenberg, 1961). However, under a speed set, no difference existed between SD and SND decision times, thus leading to the rejection of the second prediction, which was based upon the results of Greenberg's (1961) study. There were, however, several procedural differences between the present study and Greenberg's which may provide some rationale for the discrepancy.

\section{REFERENCES}

COOMBS, C. H. A theory of data. New York: Wiley, 1964

COOMBS, C. H. On the use of inconsistency of preferences in psychological measurement. J. exp. Psychol, 1958, 55, 1-7.

FALMAGNE, J. C. Stochastic models for choice reaction times with applications to experimental results. J. math. Psychol, 1965, 2, 77-125.

GREENBERG, M. C. Response latency as a test of mathematical models of preference behavior. Unpublished Ph.D. Dissertation, University of Michigan, 1962:

KELLOGG, W. N. The time of judgment in psychometric measures. Amer. $J$. Psychol, 1931, 43, 65-86.

PETRUSSIC, W. M. The relationship between stochastic and temporal dominance under an accuracy set. Psychon. Sci., 1966, 6, 373-374.

\section{NOTES}

1. This report covers a portion of the material contained in a dissertation submitted in partial fulfillment of the requirements for the Ph.D. degree at Claremont Graduate School, 1967.

2. In a two-alternative choice situation, with replications of Ss responses, the stimulus chosen: (a) more than $50 \%$ of the time is stochastically dominant (SD) and (b) less than 50\% of the time, stochastically nondominant (SND). When the proportions are equal, the stimuli are stochastically equivalent (SE). If the mean decision time for the SD choices is less than that associated with the SND choices, it is considered temporally dominant (TD); if greater, it is temporally non-dominant (TND). When the mean decision times for SD and SND choices are equal, they are temporally equivalent (TE). 